\title{
Coaching en las organizaciones: experiencia con personal administrativo en salud
}

\author{
César Mejía Chiang* \\ Kety Jáuregui Machuca**
}

Fecha de recibido: 6 de junio de 2019

Fecha de aprobado: 1 de noviembre de 2019

\begin{abstract}
Para citar este artículo: Mejía Chiang, C., \& Jáuregui Machuca, K. (2020). Coaching en las organizaciones: experiencia con personal administrativo en salud. Revista Universidad \& Empresa, 22(39), 1-26. https://doi.org/10.12804/revistas.urosario.edu.co/ empresa/a.7977
\end{abstract}

\section{Resumen}

Las organizaciones del sector salud afrontan problemas de interrelación, estrés y desmotivación del personal, derivados de la complejidad de sus labores, la estructura, los procesos y la cultura interna, debido a que el sistema de salud nacional es desarticulado y está desorganizado. Para enfrentar estos problemas, la gestión del talento humano se ha convertido en un elemento generador de valor para crear e impulsar nuevos proyectos, crear un buen clima laboral, dinamizar los servicios y generar satisfacción y fidelidad en los clientes y compromiso de los colaboradores. El coaching se ha convertido en una herramienta útil para establecer un clima laboral propicio para el desempeño productivo de los colaboradores. En este sentido, el propósito de estudio es entender cómo se implementa el coaching en el personal administrativo en una clínica peruana. Los aspectos que más contribuyeron a una implementación efectiva fueron el estilo del programa,

* Doctor en periodismo por la Universidad ceu San Pablo (España). Magíster en organización y dirección de personas por la Universidad ESAN (Perú). Magíster en periodismo y licenciado en ciencias de la comunicación por la usMP (Perú). Licenciado en educación por la Universidad Cayetano Heredia (Perú). Director del Instituto de Investigación de la Escuela de cc. cc. de la usmp. Correo electrónico: cmejiac@usmp.pe

** Doctora en administración por el IESE Business School de la Universidad de Navarra (España). Master en administración de la información por el ITESM (México). Posgrado en RR. HH. en ESADE de la Universidad Ramón Llull (España). Ingeniera industrial por la UNI (Perú). Directora de la Maestría de Organización y Dirección de Personas de la Universidad ESAN (Perú). Correo electrónico: kjauregui@esan.edu.pe 
la conexión entre el coach y el coachee, los valores y competencias del coach, la presencia de la organización y la percepción sobre la utilidad y la facilidad de aplicación.

Palabras clave: coaching; instituciones de salud; coaching en el Perú.

\title{
Coaching in Organizations: Experience with Administrative Health Personnel
}

\begin{abstract}
Health sector organizations face problems of interrelation, stress and lack of motivation of the personnel, derived from the complexity of their tasks, structure, processes, and internal culture, which are the result of a disarticulated and disorganized national health system. To face these problems, talent management has become an element that generates value to create and promote new projects, create a good work environment, boost services, and generate customer satisfaction and loyalty, and commitment from the employees. Coaching has become a useful tool to establish a favorable work environment for the productive performance of employees. In this sense, the purpose of the study is to understand how coaching is implemented in an administrative staff in a Peruvian clinic. The aspects that contributed the most to an effective implementation were the style of the program, the connection between the coach and the coachee, the coach's values and competencies, the presence of the organization, the perception of usefulness, and the ease of application.
\end{abstract}

Keywords: coaching; health institutions; coaching in Peru.

\section{Coaching nas organizações: experiência no pessoal administrativo de saúde}

\section{Resumo}

As organizações do setor da saúde afrontam problemas de interrelação, estresse e desmotivação do pessoal, derivados da complexidade de suas funções, a estrutura, os processos e a cultura interna, em um sistema de saúde nacional desarticulado e desorganizado. Para enfrentar estes problemas, a gestão do talento se tem convertido em um elemento que gera valor para criar e impulsionar novos projetos, criar um bom clima laboral, dinamizar os serviços e gerar satisfação e fidelidade nos clientes e compromisso com os colaboradores. O coaching se tem convertido em uma ferramenta útil para estabelecer um clima laboral propício para o desempenho produtivo dos colaboradores. Neste sentido, o propósito de estudo é entender como se implementa o coaching no pessoal administrativo em uma clínica peruana.

Os aspectos que mais contribuíram a uma implementação efetiva foram o estilo do programa, a conexão entre o coach e o coachee, os valores e competências do coach, a presença da organização e a percepção sobre a utilidade e a facilidade de aplicação.

Palavras-chave: coaching; instituições de saúde; coaching no Peru. 


\section{Introducción}

La prestación de servicios de salud se configura como un entorno complejo y desgastante (Junquera, 2014), donde confluyen la integridad física del paciente, la reputación profesional del médico-especialista y otros grupos como el personal técnico y administrativo, diferenciados por la escala remunerativa y la jerarquía. Dicho escenario se agudiza por pertenecer a un sector afectado por los cambios constantes en las reglamentaciones gubernamentales, la desarticulación de actores, la aparición de nuevas tecnologías y una fuerza laboral insatisfecha (Hartman \& Crow, 2002; Scott, 2002).

En la actualidad, el trabajador ha pasado de ser considerado un recurso a ser apreciado como un agente creativo, con capacidad de aprender y adaptarse a diferentes situaciones. Para ello, las organizaciones deben trabajar en estrategias para establecer un clima laboral propicio y lograr que los trabajadores estén satisfechos y puedan tener un desempeño productivo. El coaching se presenta como una técnica que puede ayudar a los líderes a lograr un buen clima laboral.

La práctica del coaching ha crecido sustancialmente desde 1990 (Neenan \& Palmer, 2001). En el campo de la salud, esta se ha centrado en el paciente a través del health coaching (Palmer, Tubbs \& Whybrow, 2003). Sin embargo, a lo largo de la última década, el coaching para los médicos, el personal técnico y el personal administrativo ha comenzado a implementarse (Sherman \& Freas, 2004). Al ser así, este estudio tiene como objetivo entender cómo se implementa el coaching en el personal administrativo de una clínica peruana.

Primero se presenta la revisión de literatura, seguida de la descripción de los criterios metodológicos empleados. Luego, se explican los hallazgos y la discusión; finalmente, se presentan las conclusiones y recomendaciones del estudio. 


\section{Revisión de la literatura}

El término coaching se deriva de un término francés definido como "conducir a una persona de un punto a otro", lo cual encaja de manera coherente con el significado actual de dicho proceso (Haas, 1992). Empero, la práctica de coaching se remonta a Sócrates, quien podría considerarse como uno de los primeros entrenadores con un método de diálogo y cuestionamiento, el cual se considera como un medio para mejorar la autoconfianza y la capacidad de razonar mediante la reflexión (Carey, Philippon \& Cummings, 2011).

El coaching ha sido estudiado desde dos perspectivas principales (Theeboom, Beersma \& van Vianen, 2013): la psicológica y la organizacional. La perspectiva psicológica involucra estudios que impactan en la depresión, la ansiedad, la autorreflexión, el estrés y la calidad de vida, entre otros (Grant 2003; Luthans \& Peterson, 2004; Green, Oades \& Grant, 2006; Spence, Cavanagh \& Grant, 2008). En la perspectiva organizacional se han realizado estudios que impactan en el desempeño, el compromiso en el trabajo, el liderazgo transformacional y la satisfacción en el trabajo, entre otros (Kilburg, 1996; Bozer \& Sarros, 2012; Cerni, Curtis \& Colmar, 2010).

En las organizaciones, el coaching se ha centrado en resolver problemas de habilidades y actitudes de los ejecutivos; actualmente también se está enfocando en preparar a otros colaboradores para avanzar profesionalmente y lograr una mejora continua (Bono et al., 2009; Giglio, Diamante \& Urban, 1998). Dentro de esta perspectiva, Renton (2010) reconoce variables constantes del coaching que lo muestran como un proceso dirigido a objetivos y resultados, que facilita el cambio voluntario de una persona a través del aprendizaje, produce crecimiento personal, destraba el potencial y maximiza el rendimiento laboral. Así mismo, genera una nueva forma de percibir, sentir y descubrir respuestas a problemas para conducirse en determinadas situaciones, contribuyendo así a aumentar la eficacia organizacional. Zeus y Skiffington (2002) mencionan que el coaching recibe una marcada influencia de la teoría de aprendizaje.

Las definiciones de coaching varían según las escuelas (Ortiz de Zárate, 2010). La escuela norteamericana, fundada por Thomas Leonard, quien creó la International Coach Federation (ICF) y la International Association of Coaching (IAC), enfoca el rol del coach en 
la escucha y la ayuda en la organización de las prioridades del coachee, actuando como guía en las decisiones que tomen. La escuela europea, a partir de Timothy Gallwey y John Whitmore (2005), hace referencia al método de hacer preguntas para la exploración y descubrimiento de creencias que resulten en el logro de objetivos, así como de liberar el potencial de una persona para mejorar su desempeño. En la escuela chilena u ontológica de Fernando Flores, Rafael Echeverría y Julio Olalla, el coaching ayuda al cliente a identificar qué tipo de observador es y le ayuda a convertirse en un observador diferente de su realidad (Ortiz de Zárate, 2010).

Echeverría (2010) sostiene que el coaching ontológico se centra en comprender al ser, sumergirse en el individuo para producir un cambio interno mediante la observación y el diálogo, logrando una vivencia comunicativa en la cual surgen emociones, corporalidad y lenguaje. Gonnet (2013) lo concibe como una técnica gerencial que asume la crisis de los modelos tradicionales que se muestran inaplicables para trabajadores cuyo trabajo depende del carácter generativo de las conversaciones. En la misma línea, Bayón et al. (2006) consideran el coaching ontológico como un método de conversación establecido por el coach, que le permite desafiar respetuosamente las formas de pensar y actuar del coachee, así como su manera de relacionarse con los demás con el fin de facilitarle el acceso a resultados extraordinarios.

\subsection{Factores que intervienen en la implementación del coaching}

Sovik (2017) afirma en su estudio que en la implementación del coaching organizacional intervienen los siguientes factores: a) el apoyo de la alta dirección, b) el contar con proveedores competentes, c) la cultura de la organización, d) el involucrar a los futuros participantes en la visión de los objetivos del programa y en la toma de decisiones relativas a la implementación, e) el contar con champions convencidos del programa en cada área de implementación y f) el nivel de liderazgo para enfrentar los desafíos de la implementación y generar un clima de trabajo constructivo. 


\subsubsection{Estilo de liderazgo del coaching}

Con respecto al estilo de liderazgo de las organizaciones, Hernández Hernández (2008) subraya que el coaching analiza opciones y ofrece pautas de acción, buscando involucrar al coachee en la determinación de objetivos y el fortalecimiento de su liderazgo, mas no una demarcación unilateral sobre lo que se debe hacer. El éxito de las sesiones recae en el coachee (Angel \& Amar, 2007). El coach basa su accionar en torno a las relaciones, evoca respuestas en el individuo y tiene un acercamiento más personal, a partir de las necesidades, valores y propósitos del individuo; en suma, se trata de un enfoque holístico. El coachee es quien establece la agenda de acuerdo con sus necesidades y disponibilidad, puesto que la intervención es personalizada, orientada a potenciar la motivación intrínseca y un cambio de conducta sostenido (Zeus \& Skiffington, 2002).

En una mirada ontológica, el coach ayuda a su coachee a identificar qué tipo de observador es para resolver los obstáculos que afectan el desempeño y afrontar eficazmente las situaciones diarias (Echeverría, 2010).

\subsubsection{Competencias del coach}

Con referencia a la conexión existente entre el coach y el coachee, Keil et al. (1996) mencionan la importancia de los niveles de confianza para obtener la apertura del participante hacia el cambio, así como la voluntad de ser influenciados.

Los valores y las competencias del coach son abordados por Giglio, Diamante y Urban (1998), quienes resaltan la importancia de la objetividad, la empatía y la curiosidad pues estas impactarán la relación entre el coach y el coachee. Por su parte, Granko, Morton y Schaafsma (2013) resaltan que la selección del coach no puede verse limitada por un tema presupuestal o de competencia en el mercado, sino que es importante considerar la calidad de este, que se refleja en su experiencia, nivel de calificación relevante, estilo de coaching y uso de recursos durante el proceso. Otras características relevantes del coach hacen referencia a su percepción aguda, la diplomacia, el buen juicio y la capacidad de superar conflictos con integridad (Kowalski \& Casper, 2007). Asimismo, en la calidad de la entrega del programa se considera la actitud del coach, sus conocimientos y habilidades, 
el uso de métodos y procedimientos interactivos que generen un entorno de confianza y faciliten la participación, y su controle del componente emocional (Sovik, 2017; Bolch, 2001; Jones \& Spooner, 2006; Schlegelmich \& Fresco, 2005).

Para que el coachee desarrolle sus competencias, el coach provee información y entrenamiento adecuados y promueve una actitud de aprendizaje a través de la formación de carácter y el fortalecimiento de la autoestima (Cardona-Soriano, 2003).

\subsubsection{Soporte y respaldo de la organización}

En relación con el soporte y respaldo de la organización, McNally y Luckens (2006) consideran que el coaching contribuye a alcanzar objetivos profesionales y personales, retener a los líderes talentosos, mejorar el desempeño individual y organizacional, así como a apoyar la planificación de la sucesión. Truijen y Woerkom (2008) propusieron algunos factores que impactan positivamente en la eficacia del coaching: contar con facilitadores del proceso y con un ambiente de soporte para lograr los resultados esperados.

\subsubsection{Soporte de los proveedores}

Hay otros factores que se relacionan con el soporte de los proveedores internos que implementan el programa: a) su percepción del programa puede influir en sus actitudes y en su motivación hacia la implementación, b) su confianza en tener las habilidades necesarias para implementar el programa, c) la fidelidad en el despliegue del programa en relación con lo planificado - contenido, frecuencia y duración-y d) contemplar las posibles adaptaciones del programa a la realidad de la organización.

Según Beattie et al. (2014) el coaching genera una mayor satisfacción y compromiso en el trabajo, así como una mejor comunicación. Adicionalmente, los autores mencionan que si la cultura organizacional no se encuentra preparada para la implementación del coaching, pueden generarse consecuencias negativas que limiten su éxito. Al respecto, Saporito (1996) afirma que la confianza en el coaching contribuye a reducir el escepticismo con respecto a su utilidad para la organización. 


\section{Metodología}

Esta investigación de carácter exploratorio y cualitativo investiga un fenómeno en profundidad y en su contexto real, con el objetivo de documentar una experiencia o entender un fenómeno desde el punto de vista de quienes lo vivieron (Yin, 2009).

\subsection{Recolección y análisis de datos}

Se seleccionó una clínica dentro de un amplio espectro de instituciones de salud de acuerdo a los siguientes criterios: a) es una clínica privada, donde existe mayor viabilidad para la implementación del coaching que en una institución pública, b) su reconocimiento en el mercado peruano la convierta en una institución representativa en su rubro, c) dio el permiso para recabar información, en tanto que la práctica del coaching es confidencial y reservada.

Se obtuvo el permiso oficial de la clínica para poder aplicar dos técnicas para la recolección de datos: la entrevista en profundidad (tabla 1) y la observación directa, dirigidas a registrar los significados y experiencias de los coachees.

Tabla 1. Ficha de cuestionario

\section{Entrevista Semiestructurada}

Fecha: ___ _ _

Código:

Edad:

Profesión:

Cargo actual:

1. ¿De qué manera se estructuraron las sesiones y actividades de coaching que usted recibió?

2. ¿Cuáles fueron los contenidos y temas desarrollados en dichas sesiones?

3. ¿Qué cualidades pudo identificar en su coach?

4. ¿Cómo describiría el ambiente y la relación que estableció con su coach?

5. ¿Qué tipo de procedimientos empleó su coach para generar el diálogo durante las sesiones?

6. ¿Qué aprendió usted tras el programa de coaching?

Ambas técnicas fueron fundamentales para el desarrollo de la investigación cualitativa, ya que permitieron registrar conceptos y percepciones de los integrantes de la muestra y, por tanto, generar segmentos de significados y categorías referidos al proceso de coaching realizado en la clínica. 
A partir de una triangulación de datos efectiva, se tuvo acceso a documentación interna y archivos referidos al programa de coaching. Con estas anotaciones, se profundizó en variables clave para comprender si su aplicación fue coherente y pertinente para el contexto de una institución de salud privada.

La muestra estuvo constituida por 6 entrevistados varones provenientes del área administrativa de la clínica, cuyas edades promediaron los 50 años (tabla 2). Los puestos de los coachees corresponden a jefaturas con más de una década laborando en la institución de salud analizada. Se adhirió voluntariamente al programa el 80\% de los participantes preseleccionados, en un muestreo por conveniencia, considerando que este tipo de programas se implementan con un número reducido de personas.

Tabla 2. Rasgos del personal administrativo entrevistado

\begin{tabular}{ccccccc}
\hline Participantes & Edad & Cargo & $\begin{array}{c}\text { Años en } \\
\text { la clínica }\end{array}$ & Profesión & $\begin{array}{c}\text { Nivel de } \\
\text { formación }\end{array}$ & $\begin{array}{c}\text { Años de } \\
\text { ejercicio } \\
\text { profesional }\end{array}$ \\
\hline E1 & 49 & Jefe de Atención al Cliente & 8 & Administrador & $\begin{array}{c}\text { Licenciatura } \\
\text { universitaria }\end{array}$ & 24 \\
\hline E2 & 56 & Jefe de Contabilidad & 12 & Contador & $\begin{array}{c}\text { Estudios de } \\
\text { maestría }\end{array}$ & 32 \\
\hline E3 & 45 & Jefe de Admisión & 6 & Administrador $\begin{array}{l}\text { Licenciatura } \\
\text { universitaria }\end{array}$ & 18 \\
\hline E4 & 47 & Jefe de Servicios Generales & 8 & Administrador & $\begin{array}{l}\text { Bachillerato } \\
\text { universitario }\end{array}$ \\
\hline E5 & 52 & Jefe de Mantenimiento & 9 & Administrador & $\begin{array}{l}\text { Licenciatura } \\
\text { universitaria }\end{array}$ \\
\hline E6 & 54 & Jefe de Gobernancia & 11 & Administrador & $\begin{array}{l}\text { Licenciatura } \\
\text { universitaria }\end{array}$ \\
\hline
\end{tabular}

\subsection{Validación de datos}

La fiabilidad de los datos cualitativos empleados en esta investigación dependió de un proceso constante de verificación de la veracidad de la información. Tal como sugiere Creswell (2009), se requirió la revisión rigurosa de las transcripciones a fin de subsanar errores generados durante la trascripción; el contraste entre las interpretaciones del investigador y la realidad propia de la fuente; repetidas visitas a la clínica para calibrar instrumentos de observación, y la aplicación de la triangulación de datos (Yin, 2009). 


\section{Resultados}

El programa de coaching tuvo un contenido ajustado a las necesidades de la clínica, que consideró la realidad ocupacional de los coachees: desbordada de ansiedad, estrés, angustia y alta carga laboral.

Los coachees reportaron que el programa produjo un impacto significativo en su forma de interactuar con los demás, así como en su concepción sobre el coaching en el ámbito corporativo. Los entrevistados destacaron cinco aspectos relevantes en la implementación: a) estilo del programa, b) conexión entre el coach y el coachee, c) valores y competencias del coach, d) presencia de la organización y e) percepción sobre la facilidad de aplicación. Dichos aspectos se configuran como categorías (tabla 3), de las cuales se desprenden subcategorías que las explican y estas, a su vez, se dividen en temas.

Tabla 3. Estructura de la información

\begin{tabular}{|c|c|c|}
\hline Categoría & Subcategoría & Temas \\
\hline \multirow{5}{*}{$\begin{array}{l}\text { Estilo del } \\
\text { programa }\end{array}$} & $\begin{array}{l}\text { Claridad en el } \\
\text { alcance }\end{array}$ & $\begin{array}{l}\text { - El objetivo fue la mejora de la comunicación para una interrelación más } \\
\text { eficaz. } \\
\text { - } \quad \text { Estuvo dirigido a jefaturas administrativas. } \\
\text { - Se establecieron claramente los roles del coach y del coachee. } \\
\text { - Se estableció la corriente ontológica del coaching. }\end{array}$ \\
\hline & $\begin{array}{l}\text { Adhesión } \\
\text { voluntaria }\end{array}$ & $\begin{array}{l}\text { - Se incorporó a participantes realmente interesados en el programa. } \\
\text { - No hubo ninguna consecuencia para quienes no deseaban adherirse al } \\
\text { programa. }\end{array}$ \\
\hline & $\begin{array}{l}\text { Enfoque } \\
\text { individualizado }\end{array}$ & $\begin{array}{l}\text { - Se superó el temor y la desconfianza por compartir temas personales. } \\
\text { - Se logró una vivencia comunicativa en la relación uno a uno entre el } \\
\text { coach y el coachee. }\end{array}$ \\
\hline & $\begin{array}{l}\text { Metodología } \\
\text { didáctica }\end{array}$ & $\begin{array}{l}\text { - Incidió en la práctica vivencial más que en técnicas pedagógicas memo- } \\
\text { rísticas. } \\
\text { - El estilo de trabajo del programa fue ágil. }\end{array}$ \\
\hline & $\begin{array}{l}\text { Manejo de la } \\
\text { libertad }\end{array}$ & $\begin{array}{l}\text { - El ritmo de las sesiones tenía una conducción compartida. } \\
\text { - El participante tuvo libertad para encontrar respuestas por sí mismo. }\end{array}$ \\
\hline
\end{tabular}




\begin{tabular}{|c|c|c|}
\hline Categoría & Subcategoría & Temas \\
\hline \multirow{3}{*}{$\begin{array}{l}\text { Conexión } \\
\text { entre el coach } \\
\text { y el coachee }\end{array}$} & $\begin{array}{l}\text { Clima de } \\
\text { confianza y } \\
\text { empatía }\end{array}$ & $\begin{array}{l}\text { - Hubo conexión real entre el coach y el coachee. } \\
\text { - Se generó una relación positiva basada en la comunicación fraternal y } \\
\text { horizontal. }\end{array}$ \\
\hline & Retroalimentación & $\begin{array}{l}\text { - Se percibió un alto grado de confidencialidad que permitió la apertura } \\
\text { a la retroalimentación. }\end{array}$ \\
\hline & & $\begin{array}{l}\text { - Se generaba una retroalimentación de ida y vuelta entre el coach y el } \\
\text { coachee }\end{array}$ \\
\hline \multirow{3}{*}{$\begin{array}{l}\text { Valores y } \\
\text { competencias } \\
\text { del coach }\end{array}$} & $\begin{array}{l}\text { Valores } \\
\text { personales }\end{array}$ & $\begin{array}{l}\text { - La amabilidad y sencillez del coach permitió una comunicación más } \\
\text { auténtica. } \\
\text { - El coach demostró compromiso con el programa y el coachee. }\end{array}$ \\
\hline & Competencias & $\begin{array}{l}\text { - La empatía a partir de observar y escuchar fue apreciada por los parti- } \\
\text { cipantes. } \\
\text { - Se observa dominio técnico: hacer preguntas y en el lenguaje verbal y } \\
\text { no verbal. }\end{array}$ \\
\hline & $\begin{array}{l}\text { Carácter } \\
\text { inspirador del } \\
\text { coach }\end{array}$ & $\begin{array}{l}\text { - El coach era coherente: aplicaba con el coachee lo que aplicaba para sí } \\
\text { mismo. } \\
\text { - El coach acompañaba al coachee en su proceso de autoconocimiento } \\
\text { y reflexión. }\end{array}$ \\
\hline \multirow{2}{*}{$\begin{array}{l}\text { Presencia } \\
\text { de la } \\
\text { organización }\end{array}$} & $\begin{array}{l}\text { Soporte de la } \\
\text { organización }\end{array}$ & $\begin{array}{l}\text { - El apoyo presencial y económico de la organización fue un factor de- } \\
\text { terminante para el programa. }\end{array}$ \\
\hline & $\begin{array}{l}\text { Respaldo de la } \\
\text { organización }\end{array}$ & $\begin{array}{l}\text { - El valor que la empresa dio al coaching para el desarrollo profesional y } \\
\text { la fidelización fue fundamental. }\end{array}$ \\
\hline \multirow{2}{*}{$\begin{array}{l}\text { Percepción } \\
\text { sobre la } \\
\text { utilidad y } \\
\text { facilidad de } \\
\text { aplicación }\end{array}$} & $\begin{array}{l}\text { Utilidad para el } \\
\text { coachee }\end{array}$ & $\begin{array}{l}\text { - El coachee reconoció la utilidad a nivel interpersonal: comunicación, } \\
\text { empatía. } \\
\text { - El coachee reconoció la utilidad a nivel personal: autoconocimiento, } \\
\text { autodominio. }\end{array}$ \\
\hline & $\begin{array}{l}\text { Facilidad de } \\
\text { aplicación en el } \\
\text { entorno laboral }\end{array}$ & $\begin{array}{l}\text { - Hubo un cambio de actitud percibido por colegas y colaboradores en } \\
\text { el corto plazo. } \\
\text { - Hubo dificultad para articular lo aprendido con la realidad en el media- } \\
\text { no plazo. }\end{array}$ \\
\hline
\end{tabular}

\subsection{Estilo del programa}

El programa tuvo un alcance claro, una adhesión voluntaria, un enfoque individual, una metodología didáctica y un manejo de la libertad. La organización decidió enfocar el coaching hacia la mejora de la comunicación dentro de las jefaturas administrativas, con la finalidad de que los participantes pudieran interrelacionarse mejor con sus superiores, colegas y subalternos. Los coaches, de nacionalidad argentina y ajenos al sector salud, provenían de la escuela latinoamericana de coaching ontológico, determinaron claramente los términos y roles del coach y coachee, para evitar ambigüedades y connotaciones jerárquicas como tutor, entrenador, mentor, pupilo o discípulo. Fue importante comunicar el 
alcance con claridad, puesto que los participantes manejaban conceptos diferentes sobre el coaching. En la opinión del entrevistado 5: "Pensaba que el coaching tenía que ver con cuestiones deportivas (...) claro, se me viene a la mente la figura del entrenador y el director técnico de un equipo de fútbol”.

Para algunos, el coaching era sinónimo de consultoría o asesoría, lo que estaría centrado específicamente en corregir sus falencias y debilidades, las cuales habrían sido detectadas durante su desenvolvimiento en el trabajo: "Me preocupé un poco cuando me hablaron que se venían los cursos de coaching (...) porque algo me faltaba o estaba haciendo algo mal (...) o a lo mejor era un reconocimiento" (Entrevistado 4).

La adhesión voluntaria al programa permitió incorporar a los participantes realmente interesados en los objetivos y beneficios del coaching, y no hubo ninguna consecuencia negativa para quienes no se sentían interesados o preparados para enrolarse en el programa.

La relación individual entre coach y coachee fue uno de los pilares que aseguraron el buen desenvolvimiento del programa, que permitieron que se superaran el temor y la desconfianza de los participantes por compartir sus dificultades personales con sus colegas.

Los coachees agradecieron el sentido didáctico de las sesiones, que no se centraron excesivamente en técnicas pedagógicas tradicionales y memorísticas. En ese sentido, se reconoció el ágil estilo de trabajo del programa, basado en el empleo acertado de casos reales de estudio: "Me sorprendió mucho que las lecturas fueran breves (...) y que todas tratasen de experiencias reales, con las que te puedas sentir identificado" (Entrevistado 2). Asimismo, el Entrevistado 3 mencionó: "Casi al momento intuí que este coach (...) buscaba ofrecerme algo más vivencial, obviando el hecho de que esto fuera un paquete individualizado". La metodología empleada en las diez sesiones del programa convenció a los participantes de la validez y pertinencia de la intervención.

Se destaca la libertad de los coachees para conducir en forma compartida el ritmo en cada sesión: 
Desde el primer día, el coach (...) me regaló algo muy importante y que creo que desde niño no había tenido oportunidad de aprovecharlo: el tiempo y la libertad de encontrar las respuestas por mí mismo, sin depender de las órdenes de arriba (Entrevistado 5).

En las sesiones, mi coach me planteaba los asuntos a abordar, me preguntaba con cuáles me gustaría comenzar (...) y encontrábamos el momento preciso para poder dialogar al respecto... no era pues imposición, era una reflexión conjunta, pero siempre guiada por mis intereses, mis preocupaciones del momento (Entrevistado 6).

\subsection{Conexión entre el coach y el coachee}

El coach y el coachee establecieron un clima de confianza y empatía que permitió una relación positiva y la canalización de una retroalimentación fluida. Hubo una conexión real entre ambos. El entrevistado 2 subrayó el nivel de conexión que había logrado con su coach: "No ponía en duda que, en muchos de sus comentarios, el coach acertaba. Otra cuestión era que yo no quería reconocerlo". Al respecto, el entrevistado 6 afirmó: "El coach iba más allá de mi explicación para hacer una metaexplicación (...) comprendiendo mis creencias (...) mis intereses (...) y como se percibían dentro de la clínica”.

Esta conexión generó una relación positiva basada en la comunicación fraternal y horizontal. Los coachees reconocieron positivamente la relación con los coaches:

Me gustó que la comunicación fuese fraternal y no instructiva (...) el coach me dijo que él no era mi "mentor" ni "maestro", ni un "juez", sino un compañero que me ayudaría (...) Pasaban las sesiones y con esta sinceridad y esa forma de dialogar yo no me sentía "acusado" y eso evitaba que me pusiese a la defensiva (Entrevistado 2).

Los coaches lograron establecer con los participantes unos lazos profundos y significativos, que desencadenaron en la creación de un espacio de entera confianza y distensión: "El feedback que regularmente recibía no solo me ayudó profesionalmente, sino que hizo posible labrar una profunda amistad con mi coach (...) He ganado un buen amigo y quizá confidente" (Entrevistado 3). Sobre el mismo tema, el entrevistado 2 mencionó: "Desde las primeras sesiones me llevé bien con mi coach (...) en un ambiente de confianza, 
mucho compañerismo y complicidad (...) eso no significa que haya sido permisivo, pero sí muy comprensivo" (Entrevistado 2).

La amistad forjada entre el coach y el coachee sirvió como estrategia para hacer más efectivo el proceso de coaching, ayudando a crear compromiso con los objetivos del programa.

La retroalimentación desarrollada durante las sesiones pudo canalizarse convenientemente a través del alto grado de confidencialidad. Los participantes concordaron que al tratarse de un coach externo no solo hubo mayor profesionalismo, sino también discreción e imparcialidad durante el diálogo:

Cuando en la inducción nos mostraron los perfiles de los coaches me dio bastante alivio que estos fueran extranjeros (...) Con un coach externo había más privacidad, que aseguraba todas las garantías de confidencialidad que habíamos firmado previo a esto (Entrevistado 4).

Se llegó a generar una retroalimentación de ida y vuelta entre el coach y el coachee.

Cada sesión tenía [un] espacio para cuestionar al propio coach con preguntas que aparte de hacer más horizontal la conversación, (...) te impulsaban a mirarte a ti mismo y evaluar tu propio rendimiento (Entrevistado 5).

Asimismo, el Entrevistado 1 mencionó:

Me gustó el hecho que el coach también compartiese algunos de sus problemas, inquietudes, curiosidades y demás. Eso ayudaba a que el coaching no [fuera] un confesionario donde yo sería siempre la supuesta víctima (...) Con ese intercambio, comprendí que muchos problemas eran comunes y humanos y que no debería sentirme culpable de no resolverlos tan rápido.

\subsection{Valores y competencias del coach}

Cualidades como la amabilidad y la sencillez resaltaron como signos preponderantes de los coaches, que permitieron una comunicación más auténtica, que acrecentó aún más la 
confianza y un ambiente de apertura idóneo para la retroalimentación, el aprendizaje y el crecimiento personal:

Al revisar la hoja de vida, las publicaciones y los grados académicos de mi coach pensé encontrarme con un erudito alejado de la realidad de una organización y que solo tenía contacto con los libros (...) pero cuando lo conocí me pareció la persona más amable y sencilla que uno pueda imaginar (Entrevistado 4).

Los participantes coinciden en que los coaches mostraron un compromiso con las premisas generales del programa y con los propios coachees, ajustándose a las necesidades de estos últimos, fueron tolerantes, pacientes y respetuosos mientras transcurrían las sesiones:

En las sesiones [sentí] una preocupación de que yo mejorara, como si fuera el consejo de un amigo de años, como del colegio o de la universidad... al igual que con ellos, me hacía sentir cómodo (...) era como reencontrarte o redescubrir a un viejo amigo (Entrevistado 5).

Las capacidadades de observar y escuchar del coach fueron aspectos apreciados por los participantes e interpretados como una demostración de la experiencia y experticia del especialista en este campo:

Mientras yo hablaba, mi coach observaba, tomaba algunas notas, pero manteniendo contacto visual (...) Cuando le tocaba a él hablar, dosificaba el volumen de su voz, cuidando no interrumpirme (...) siempre sentí esa disposición de querer comprender y ponerse en el otro lado (Entrevistado 4).

Entre las cualidades que exhibieron los coaches, destacaron la escucha activa, la formulación hábil de preguntas, la empatía, el dominio del lenguaje verbal y no verbal, el humor y el conocimiento profundo para desarrollar la intervención.

Por último, pero no menos importante, se subraya el carácter inspirador del coach. Como guía y facilitador, ayudó a los participantes a alcanzar la autoobservación y la autorreflexión, así como una comprensión realista de sus puntos fuertes y débiles, a fin 
de proyectarse hacia el futuro. Se convirtieron en referentes dignos de emulación, que inspiraron aspectos positivos en los coachees.

En esa línea, los coachees valoraron las actitudes y posturas que adoptaron sus respectivos coaches durante las sesiones e incluso algunos de ellos anhelaron replicarlas en su rutina laboral, lo cual refleja su convencimiento en el coaching y en su efectividad. El entrevistado 2 mencionó: "Lo que más aprendí de mi coach fue aceptar la crítica y evitar estar siempre a la defensiva cuando me siento atacado (...) Eso se logra con una comunicación razonable (...) Gracias a mi coach pude hacer ese cambio”. Según el entrevistado 5:

El coach me dio esa sensación de coherencia del decir y hacer (...) desde que lo escuché me imaginé siempre que eso era verdad porque parecía que le funcionaba (...) No derrochaba arrogancia, sino bastante alegría, ese carisma despreocupado y libre que ves pocas veces en la gente (...). Es admirable.

\subsection{Presencia de la organización}

En determinados pasajes de las entrevistas realizadas, emergió la figura de la organización como ente presente en la carrera profesional de los coachees, así como factor determinante para el éxito del programa:

He participado en otros talleres y seminarios en los últimos años y creo que existe una línea común entre estos: la idea de descubrir y aprovechar el potencial de las personas, de poder conocerlas mejor, comprenderlas en toda su dimensión y hacerlas conscientes del papel que realizan dentro de la clínica... la razón por la cual recibimos este tipo de capacitación es para identificarnos más con la clínica, que necesita personal de confianza, leal, que no se irá a la competencia a la primera oportunidad que se le presente (Entrevistado 1).

\subsection{Percepción sobre utilidad y facilidad de aplicación}

Al concluir con las diez sesiones programadas, los participantes del área administrativa de la clínica reconocieron que el programa fue útil en muchos aspectos que iban más allá 
de la comunicación: autoconocimiento, autodominio y empatía, entre otros. Esto amplió y enriqueció sus concepciones acerca del coaching:

Aprendí a comunicarme de la mejor manera posible, sin perder mi estilo ni identidad (...) no sonar como una máquina sin criterio, pero también para conocerme a mí mismo, ser consciente de mis limitaciones y descubrir todo mi potencial a favor de la clínica (Entrevistado 3).

Sin embargo, surgió una brecha entre las sesiones de coaching y el entorno laboral, lo cual fue un punto sensible en los entrevistados. Si bien pareciera que se produjo, al inicio, un tránsito fluido y natural de lo impartido, en el que los colegas y colaboradores eran los aparentes beneficiados, con el pasar de los meses, los coachees se enfrentaron con una realidad organizacional que les impedía avanzar y articular lo aprendido, que resultó en una especie de sensación fatalista que dictaba un anticipado fracaso de toda iniciativa ambiciosa de cambio:

Muchos se enteraron que estaba llevando este programa los fines de semana y se sorprendieron del cambio de mi carácter, de lo temperamental que era hace semanas atrás y ahora (ya no tanto). Uno de mis asistentes me comentó que no lo haga "muy evidente", porque después nadie confiaría en mí y no me tomarían en serio. Él y otros analistas esperaban que volviese a mi estado "normal" y que todo fuera como antes (Entrevistado 6).

Algunos participantes compartieron críticas hacia las estructuras y el comportamiento vigente dentro de la institución de salud, la cual puede, por un lado, apoyar este tipo de cursos e iniciativas, pero que en la realidad diaria puede estar actuando paradójicamente al contrario, socavándo los objetivos primigenios del programa de coaching:

Ojalá que todo lo que he aprendido en este curso no se diluya como ha sucedido en otras ocasiones (...) $\mathrm{Ha}[\mathrm{n}]$ pasado ya un par de meses y me encuentro con que mi entorno laboral sigue siendo tan caótico, frustrante, lleno de resentimientos y problemas de comunicación, incluso con las mismas secciones de administración (...) Hay mucho espíritu competitivo, de "bailar con tu propio pañuelo" y echarle la culpa a los 
demás para librarte del castigo. Nadie quiere asumir la responsabilidad, especialmente cuando las cosas salen mal (Entrevistado 4).

Sería muy injusto decirte que el coaching ha sido algo "cosmético", porque he asimilado mucho de su lógica y las sugerencias del coach han sido muy valiosas para mí (...) El coaching podría ser esa gran ola que viene con ímpetu y fuerza para cambiar las cosas pero que irremediablemente se choca contra una costa rocosa, afilada que no cambia, que se contenta con escuchar el vaivén del agua, sin ceder nada (...) No quiero pecar de pesimista, pero falta todavía mucho tiempo y mucho coaching para crear conciencia y cambio aquí (Entrevistado 2).

\section{Discusión}

\subsection{Estilo del programa}

Con relación a la claridad del alcance, como se ha podido contemplar en el estudio, la perspectiva ontológica contribuyó a fortalecer la relación entre el coach y el coachee, en tanto implica que el coach desarrolle un interés genuino por comprender la realidad inmediata del coachee, tal como lo ha planteado Echeverría (2010). Asimismo, en concordancia con lo que afirman Bayón et al. (2006), en lugar de incidir excesivamente en sus problemas laborales, los coaches ontológicos establecieron, sin prescripciones severas o normas inflexibles, una filosofía de contacto para adentrarse en la cotidianidad y creencias de los participantes para que estos se den cuenta de los hábitos que perjudican su rendimiento en la clínica: específicamente, su forma de comunicarse y relacionarse con los demás.

Es importante mencionar que fue una elección de la clínica que los coaches fueran externos, lo cual concuerda con el consenso de diversos autores rescatado por Carey, Philippon y Cummings (2011) sobre la predominancia del impacto del coaching externo versus el interno. 
En cuanto a la adhesión, Renton (2010) reconoce al coaching como un proceso dirigido a los objetivos y a los resultados, que facilita el cambio de una persona en forma voluntaria a través del aprendizaje. Las evidencias del estudio confirman lo mencionado en la literatura, en tanto que se incorporó a participantes realmente interesados en el programa y no hubo ninguna consecuencia para quienes no deseaban adherirse a él.

Con un enfoque individualizado se logró una vivencia comunicativa en la relación uno a uno entre el coach y el coachee, en consonancia con lo que afirma Echeverría (2010) en cuanto a que el coaching se sumerge en el individuo para producir un cambio interno.

Frente a la metodología empleada en el programa, esta se caracterizó por ser dinámica y con alto sentido de libertad y alta participación del coachee, tal como se menciona en la literatura sobre el involucramiento del coachee en la determinación de objetivos y el fortalecimiento de su liderazgo, sin una demarcación unilateral sobre lo que se debe hacer (Hernández Hernández, 2008; Sovik, 2017), así como la participación activa en el desarrollo de la agenda (Zeus \& Skiffington, 2002). La libertad también fue manejada para que el participante encontrara respuestas por sí mismo, es el coachee quien identifica y afronta los obstáculos de su propio desempeño (Echeverría, 2010).

\subsection{Conexión entre el coach y el coachee}

El estudio revela que se generó una conexión real entre el coach y el coachee, lo cual contribuyó a la construcción de una relación positiva basada en la comunicación fraternal y horizontal. Lo anterior se traduce en el nivel de confianza generado entre ambas partes. Esta realidad coincide con Keil et al. (1996) sobre la importancia de la confianza para obtener la apertura voluntaria del participante al cambio.

\subsection{Valores y competencias del coach}

Cabe destacar que la calidad de los coaches impresionó positivamente a los participantes, quienes observaron sus valores personales de amabilidad, sencillez y compromiso con el programa, lo cual facilitó la interacción, en concordancia con lo mencionado por Kowalski y Casper (2007) acerca de la integridad del coach. 
Los participantes, asimismo, resaltaron las competencias de empatía, comunicación y dominio técnico, lo cual coincide con el estudio de Giglio, Diamante y Urban (1998), quienes resaltan la importancia de la empatía por parte del coach, así como el estudio de Granko, Morton y Schaafsma (2013), quienes sostienen la importancia de elegir a un coach ejecutivo con una adecuada experiencia y capacitación y un adecuado estilo de coaching y recursos que pueda ofrecer.

Por último, los coachees destacaron el carácter inspirador de los coaches: ambos demostraron coherencia entre su discurso y su acción, y disposición de acompañamiento al coachee en su proceso de autoconocimiento y reflexión (Kowalski \& Casper, 2007).

\subsection{Presencia de la organización}

Como parte del soporte que brinda la organización al programa, el apoyo presencial de la organización fue un tema determinante, así como el apoyo económico, que propiciaron un ambiente de soporte para el coaching efectivo (Truijen \& Woerkom, 2008).

La fluidez del programa de coaching pudo concretarse cuando los responsables de la rama de capacitación y desarrollo procuraron diseñar, acorde con la filosofía del coaching ontológico, un clima de enseñanza-aprendizaje que inspirase confianza, empatía y comprensión de la realidad laboral de los participantes.

Con relación al respaldo, la alta dirección de la organización tiene la convicción de la importancia del coaching para desarrollar el capital humano, que está en concordancia con Renton (2010) y Sovik (2017). Asimismo, el respaldo al programa buscaba contribuir con la fidelización del colaborador, respaldando la afirmación de McNally y Lukens (2006) sobre el impacto del coaching en la mejora del desempeño de los líderes, la retención de líderes y el soporte al plan de sucesión.

\subsection{Percepción sobre utilidad y facilidad de aplicación}

Luego del estudio, queda clara la utilidad del programa para el participante tanto a nivel personal como interpersonal, puesto que este genera una mayor satisfacción y compromiso con el trabajo, así como una mejor comunicación (Beattie et al. 2016). 
Sin embargo, aunque los colaboradores de la clínica identificaron un cambio de actitud en los participantes del programa, luego reconocieron (junto con los coachees) la dificultad para articular lo aprendido con la práctica en el mediano plazo.

En los participantes persisten aún las creencias de que los colaboradores ponen trabas a la sinergia y no buscan asumir responsabilidades ni dejarse guiar por el nuevo estilo de liderazgo que pretenden aplicar. Por ese motivo, comienzan a cuestionarse si el cambio que han experimentado, a través del coaching, es sostenible o solo ocasional o restringido en el ámbito personal/extralaboral. Con relación a este cuestionamiento, Saporito (1996) afirma que la confianza en el coaching contribuye a reducir el escepticismo con respecto a su utilidad.

Por tanto, se aprecia la urgencia por formalizar las prácticas del coaching hacia un proceso estructurado de desarrollo, seguimiento y medición de los resultados y las competencias del negocio (Lasala, 2003), a fin de que adquiera una naturaleza más técnica y cuantitativa (Cardona \& García-Lombardía, 2005).

Un punto relevante a considerar, es si la cultura de la organización se encuentra preparada para el coaching (Beattie et al., 2016), pues una implementación prematura podría ser perjudicial para su éxito.

\section{Conclusiones y recomendaciones}

\section{Conclusiones}

Los aspectos relevantes evidenciados en el estudio corresponden al estilo del programa: la conexión entre el coach y el coachee, las competencias del coach, la presencia de la organización y la percepción sobre la facilidad de la aplicación.

En el estilo del programa resalta la elección del coaching ontológico y la práctica vivencial en cada una de las sesiones presenciales. Además, el tratamiento ético y reservado 
de la información obtenida por el coach contribuyó en la aceptación del programa por parte de los participantes. Del mismo modo, la naturaleza voluntaria del programa pudo reforzar lo anterior, ya que esta característica optativa no se da en el resto de cursos y talleres desarrollados en la clínica.

En la conexión entre el coach y el coachee, destaca la dinámica de retroalimentación con un modelo dialógico, basada en un proceso individualizado que desarrolló nuevos escenarios de mejora a partir del autoconocimiento, el autodominio, la capacidad de reflexión y análisis para revitalizar la motivación, la autoestima y satisfacción personal, el liderazgo y la adaptación al cambio. Asimismo, destaca la creación y el mantenimiento de un clima de confianza y libertad.

Los valores y las competencias del coach enfatizan el carácter inspiracional irradiado, a partir de su escucha activa y empatía. El coach se mostró ante los participantes como una figura orientadora y de guía, no como un instructor o un mentor que tenía, de antemano, todas las respuestas a sus problemas. Precisamente, el perfil abierto, receptivo y dialogante de los coaches sirvió para que los participantes pudiesen construir sus propias alternativas de solución, sin que la visión del coach prevaleciera necesariamente.

La presencia de la organización se ha presentado como fundamental para la implementación eficaz del programa de coaching, considerándose tanto la capacidad de brindar soporte a la gestión, como el respaldo desde la alta dirección al proceso, demostrando una valoración por el desarrollo profesional de los participantes y la fidelización de los mismos hacia la organización.

La conexión entre el aprendizaje y la práctica del coaching ha sido compleja, debido a la limitada receptividad del entorno organizacional a los cambios de los egresados del programa, lo cual puede llevar a que estos se desalienten y desconfíen de los principios del coaching al encontrarse con una realidad que no les permite aplicar, con plenitud, lo aprendido. Los coachees se sienten limitados en practicar las técnicas de comunicación centradas en abordar el cultivo de relaciones laborales fructíferas y menos conflictivas; el fortalecimiento de sentimientos de identificación del personal subalterno con los valores organizacionales; el consenso y la flexibilización en los procesos, así como la integración y confianza entre el personal asistencialista, administrativo y de recursos humanos. 
A pesar de los inconvenientes, la implementación del coaching en sí misma evidencia un primer avance significativo en la manera de concebir la administración contemporánea, que tiene en cuenta una serie de componentes emocionales para afrontar cuadros como estrés laboral, depresión, actitudes de abandono y resignación. A partir de acciones como esta, se logrará vislumbrar líneas de carrera, planes de sucesión y posibilidades de crecimiento para desarrollar mejor el potencial de los colaboradores.

\section{Recomendaciones}

Las recomendaciones giran en torno al rol del área de recursos humanos en el monitoreo del programa, en tanto fue un punto de mejora identificado en el estudio, pues no se logró medir con claridad los resultados; hubo indiferencia en el resto de los miembros de la organización por los cambios generados con relación a los cambios esperados.

Por lo anterior, se tendrían que generar mecanismos de monitoreo de avances adicionales, complementarios a la autorregulación de los participantes, a fin de reducir el riesgo de que se convierta en errática.

El programa implementado podría configurarse como un gestor versátil de conocimiento, capaz de difundir una cultura organizacional que inspire confianza entre los coachees y colaboradores, ayudando a canalizar convenientemente las etapas de inducción, socialización y desvinculación profesional.

Si se desea reposicionar el coaching como una necesidad estratégica dentro de la clínica, se tienen que encuadrar los mecanismos y procedimientos de seguimiento para evaluar cuantitativamente los resultados del programa, a fin de que el coaching no sea considerado un taller de distensión que se nutre subrepticiamente de la insatisfacción de colaboradores poco comprometidos con la organización o con muchos problemas personales que interfieren en demasía con su trabajo. 


\section{Referencias}

Angel, P., \& Amar, P. (2007). Guía práctica del coaching. Barcelona: Paidós.

Bayón, F., Cubeiro, J., Romo, M., \& Sáinz, J. (2006). Coaching realmente, un enfoque europeo y empresarial. Madrid: Pearson Prentice Hall.

Beattie, R., Kim, S., Hagen, M., Egan, T., Ellinger, A., \& Hamlin, R. (2014). Managerial coaching: A review of the empirical literature and development of a model to guide future practice. Advances in Developing Human Resources, 16(2), 184-201.

Bolch, M. (2001). Proactive coaching. Training, 38(5), 58-66.

Bono, J., Purvanova, R., Towler, A., \& Peterson, D. (2009). A survey of executive coaching practices. Personnel Psychology, 62(2), 361-404.

Bozer, G., \& Sarros, J. (2012). Examining the effectiveness of executive coaching on coaches' performance in the Israeli context. International Journal of Evidence Based Coaching and Mentoring, 10, 14-32.

Cardona-Soriano, P. (2003). El coaching en el desarrollo de las competencias profesionales. En J. M. Casado, M. Vilallonga \& F. Alcaide, Coaching directivo: Desarrollando el liderazgo (pp. 147-166). Barcelona: Ariel.

Cardona, P., \& García-Lombardía, P. (2005) Cómo desarrollar las competencias de liderazgo. Pamplona: EUNSA.

Carey, W., Philippon, D., \& Cummings, G. (2011). Coaching models for leadership development: An integrative review. Journal of Leadership Studies, 5(1), 51-69.

Cerni, T., Curtis, G. J., \& Colmar, S. H. (2010). Executive coaching can enhance transformational leadership. International Coaching Psychology Review, 5, 81-85.

Creswell, J. (2009). Research Design. Qualitative, Quantitative, and Mixed Methods Approaches. California: Sage Publications.

Echeverría, R. (2010). Colofón al arte de soplar brasas. Sobre el coaching ontológico. En L. Wolk, Coaching. El arte de soplar brasas (pp. 203-222). Buenos Aires: Gran Aldea Editores. Recuperado de http://escuelainternacionaldecoaching.com/downloads/MATERIAL/ Coaching\%20-\%20El\%20Arte\%20de\%20Soplar\%20Brasas.pdf

Giglio, L., Diamante, Y., \& Urban, J. (1998). Coaching a leader: Leveraging change at the top. Journal of Management, 17(2), 93-105.

Gonnet, J. (2013). El discurso contemporáneo del management. El caso del coaching ontológico. Estudios Gerenciales, 29(126), 86-91. 
Granko, R., Morton, C., \& Schaafsma, K. (2013). Role of executive coaching in pharmacy management. American Journal Health-System Pharm, 70(1), 1883-1885.

Grant, A. (2003). The impact of life coaching on goal attainment, metacognition and mental health. Social Behavior and Personality, 31, 253-264.

Grant, A. (2007). Enhancing coach skills and emotional intelligence through training. Industrial and Commercial Training, 39(5), 257-266.

Green, L., Oades, L., \& Grant, A. (2006). Cognitive-behavioural, solution-focused life coaching: Enhancing goal striving, well-being and hope. Journal of Positive Psychology, 1, 142-149.

Haas, S. (1992). Coaching: Developing key players. Journal of Nursing Administration, 22(6), 54-58.

Hartman, S., \& Crow, S. (2002). Executive development in healthcare during times of turbulence: Top management perceptions and recommendations. Journal of Management in Medicine, 16(5), 359-370.

Hernández Hernández, K. (2008). Plan de recursos bumanos. Aplicación de un proceso de coaching a los supervisores de sGs en provincias para contribuir a la disminución de la rotación de personal. (Tesis de licenciatura, Lima, Universidad de San Martín de Porres).

Jones, G., \& Spooner, K. (2006). Coaching high achievers. Consulting Psychology, 58(1), 40-50.

Junquera, F. (2014). Coaching, ciencia y salud. Madrid: Lid Editorial.

Keil, F., Rimmer, E., Williams, K., \& Doyle, M. (1996). Coaching at the top. Consulting Psychology Journal: Practice and Research, 48(2), 67-77.

Kilburg, R. (1996). Toward a conceptual understanding and definition of executive coaching. Consulting Psychology Journal: Practice and Research, 48, 134-144.

Kowalski, K., \& Casper. (2007). The coaching process: An effective tool for professional development. Nursing Administration Quarterly, 31(2), 171-179.

Lasala, K. (2003) Técnicas de entrenamiento deportivas para el desarrollo de colaboradores. En M. Vilallonga (Coord.), Coaching directivo: desarrollando el liderazgo. Fundamentos y práctica del coaching (pp. 167-202). Barcelona: Ariel.

Luthans, F., \& Peterson, S. (2004). 360-degree feedback with systematic coaching: Empirical analysis suggests a winning combination. Human Resource Management, 42, 243-256.

McNally, K., \& Lukens, R. (2006). Leadership development: An external-internal coaching process. Journal of Nursing Administration, 36(3), 155-161.

Neenan, M., \& Palmer, S. (2001). Cognitive behavioural coaching. Stress News, 13(3), 15-18. 
Ortiz de Zárate, M. (2010). Psicología y coaching: marco general, las diferentes escuelas. Capital Humano, 243, 56-68.

Palmer, S., Tubbs, I., \& Whybrow, A. (2003). Health coaching to facilitate the promotion of healthy behaviour and achievement of health-related goals. International Journal of Health Promotion \& Education, 41(3), 91-93.

Renton, J. (2010). Coaching y mentoring. Técnicas para el desarrollo de los recursos humanos. Lima: The Economist, Luppa Solutions.

Saporito, T. (1996). Business-linked executive development: Coaching senior executives. Consulting Psychology Journal: Practice and Research, 48(2), 96-103.

Schlegelmich, A., \& Fresco, D. (2005). Emotion-focused therapy: Coaching clients to work through their feelings. Counselling Psychology Quarterly, 18(3), 225-226.

Scott, G. (2002). Coach, challenge, lead: Developing an indispensable management team. Healthcare Executive, $17(6)$, 16-20.

Sherman, S., \& Freas, A. (2004). The wild west of executive coaching. Harvard Business Review, 82(11), 82-90.

Sovik, M. (2017). Evaluating the implementation of the empowering coaching program in Norway (Tesis doctoral, Noruega, Universidad de Bergen). Recuperado de http://dspace. uib.no/bitstream/handle/1956/15708/dr-thesis-2017-Margaret-L-S\%C3\%B8vik.pdf?sequence=1\&isAllowed=y

Spence, G., Cavanagh, M., \& Grant, A. (2008). The integration of mindfulness training and health coaching: An exploratory study. Coaching: An International Journal of Theory, Research and Practice, 1, 145-163.

Theeboom, T., Beersma, B., \& Van Vianen, A. (2013). Does coaching work? A meta-analysis on the effects of coaching on individual level outcomes in an organizational context. The Journal of Positive Psychology, 9(1), 1-18.

Truijen, K., \& Woerkom, M. (2008). The pitfalls of collegial coaching. Journal of Workplace Learning, 29(5), 316-326.

Whitmore, J. (2005). Coaching: el método para mejorar el rendimiento de las personas. Barcelona: Paidós.

Yin, R. (2009). Case study research. Design and methods. California: Sage Publications.

Zeus, P., \& Skiffington, S. (2002). Guía completa de coaching en el trabajo. Madrid: McGraw-Hill. 